\title{
Huge uterine myoma as a cause of thromboemobolic stroke
}

\author{
Journal of \\ Neurocritical \\ Care

\section{CASE REPORT} \\ Received: September 2, 2019 \\ Revised: November 8, 2019 \\ Accepted: November 12, 2019 \\ Corresponding Author: \\ Dong Hoon Shin, MD \\ Department of Neurology, Gachon \\ University Gil Medical Center, 21 \\ Namdong-daero 774beon-gil, \\ Namdong-gu, Incheon 21565, Republic \\ of Korea \\ Tel: +82-32-460-3346 \\ Fax: +83-32-460-3344 \\ E-mail:dr.donghoon.shin@gmail.com
}

\author{
Hyun Joon Lee, MD; Dong Hoon Shin, MD \\ Department of Neurology, Gachon University Gil Medical Center, Incheon, Republic of Korea
}

Background: Embolic stroke undetermined source (ESUS), which is defined as nonlacunar infarction in the absence of cardioembolic sources, proximal artery stenosis excluded by echocardiogram, holter monitoring and vascular images, is reported to account for $9 \%$ to $25 \%$ of ischemic stroke. Because the source of embolism remains unclear, it is an important task to find the etiology for secondary prevention of stroke recurrent.

Case Report: We report a case of uterine myoma found in an embolic stroke patient with incidentally found a huge uterine myoma and related deep vein thrombosis.

Conclusion: Uterine myoma in a middle-aged woman can be thought to be the etiological cause that can contributor to deep vein thrombosis, and it is necessary to pay attention as the etiology of ESUS.

Keywords: Embolic stroke undetermined source; Embolic stroke; Myoma

\section{INTRODUCTION}

Embolic stroke undetermined source (ESUS) proposed by an International Working Group of Neurologists is a new definition to reassess the term cryptogenic stroke and change the vague defined entity of cryptogenic stroke to more clinically useful for future secondary prevention trials [1]. ESUS is defined as a nonlacunar infarct without large artery stenosis or cardioembolic sources, which is established by a stepwise diagnostic work-up. It is hypothesized that anticoagulation therapy is more efficacious than antiplatelet therapy for secondary prevention in ESUS patients [2].

Understanding the etiology of cerebral infarction is necessary to determine the prognosis or treatment of the patients. There are very rare cases of deep vein thrombosis and pulmonary thromboembolism caused by uterine myoma. In addition, there are very few reports of systemic embolism such as stroke caused by the presence of patent foramen ovale (PFO) in patients in these cases [3]. We describe a case of top of basilar syndrome in a young female patient with deep venous thrombosis due to venous compression by large uterine myoma.

(C) 2019 The Korean Neurocritical Care Society

This is an Open Access article distributed under the terms of the Creative Commons Attribution Non-Commercial License (http://creativecommons.org/licenses/by-nc/4.0/) which permits unrestricted noncommercial use, distribution, and reproduction in any medium, provided the original work is properly cited. 


\section{CASE REPORT}

A 43-year-old woman came to our hospital with loss of consciousness that occurred in the house just before hospital visit. She arrived in the emergency room 45 minutes after symptom occurred. She had no past medical history and was neither smoking nor drinking alcohol. At the time of admission, the vital sign showed a blood pressure of $119 / 66 \mathrm{~mm} \mathrm{Hg}$, a heart rate of $76 / \mathrm{min}$, a respiratory rate of $24 / \mathrm{min}$, and a body temperature of $36.2^{\circ} \mathrm{C}$. In neurological examinations, level of consciousness was stuporous, and higher cortical function could not be assessed. Both pupils were dilated $5 \mathrm{~mm} / 7 \mathrm{~mm}$, and no light reflex was seen. The patient had no motor weakness, deep tendon reflex was normoreflexia, no pathologic reflex shown. In the emergency room, brain computed tomography (CT), CT angiography, and CT perfusion were performed. Although there were no remarkable findings on brain CT (Fig. 1A) and no stenosis or occlusion on brain CT angiography (Fig. 1E), brain CT perfusion showed mildly decreased cerebral blood flow and cerebral blood volume in left midbrain (Fig. 1B$1 D)$. We started to infuse tissue plasminogen activator (tPA) intravenously after 1 hour and 40 minutes from symptom onset. Because there was no occluded vessel on brain CT angiography, we did not consider further treatment like mechanical thrombectomy. In laboratory test, protein $\mathrm{C}$ activity was decreased to $31 \%$ (normal range, $55 \%$ to $123 \%$ ), cancer antigen-125 (CA-125) was mildly elevated to $39.5 \mathrm{U} / \mathrm{mL}$ (normal range, <35). However, there were no more abnormal results in complete blood count, lipid profiles, hemoglobin A1c, thyroid function, liver, and renal function. Other blood tests related to hypercoagulability and vasculopathies including protein S activity, antithrombin III, prothrombin, antiphospholipid antibody, anticardiolipin antibody, lupus anticoagulant, antinuclear antibody, anti-beta2-glycoprotein 1 (GP 1) antibody showed negative. In magnetic resonance imaging including diffusion weighted image (DWI) and angiography, which was performed after 24 hours from tPA infusion, DWI showed hyperintensity lesions at the right side of the cerebellum, at the bilaterally thalamus, midbrain, pons (Fig. 2A) and MR angiography showed no stenosis or atherosclerotic changes (Fig. 2B). The patient admitted to intensive care unit and took anti platelet (aspirin $100 \mathrm{mg}$ / day, clopidogrel $75 \mathrm{mg}$ /day), 3-hydroxy-3-methylglutaryl coenzyme A (HMG-CoA) reductase inhibitor (atorvastatin $20 \mathrm{mg}$ ) agents for secondary prevention. Her 24 hours holter monitoring and transthoracic echocardiography were all normal. Because CA125 was elevated in laboratory findings, abdominal pelvis CT was performed to find the presence of pelvic mass. A large sized uterine myoma of $7.5 \mathrm{~cm}$ was found (Fig. 3A). Femoral CT angiography was also performed, there were multiple thrombus was found in both common iliac vein and femoral vein (Fig. 3B). For deep vein
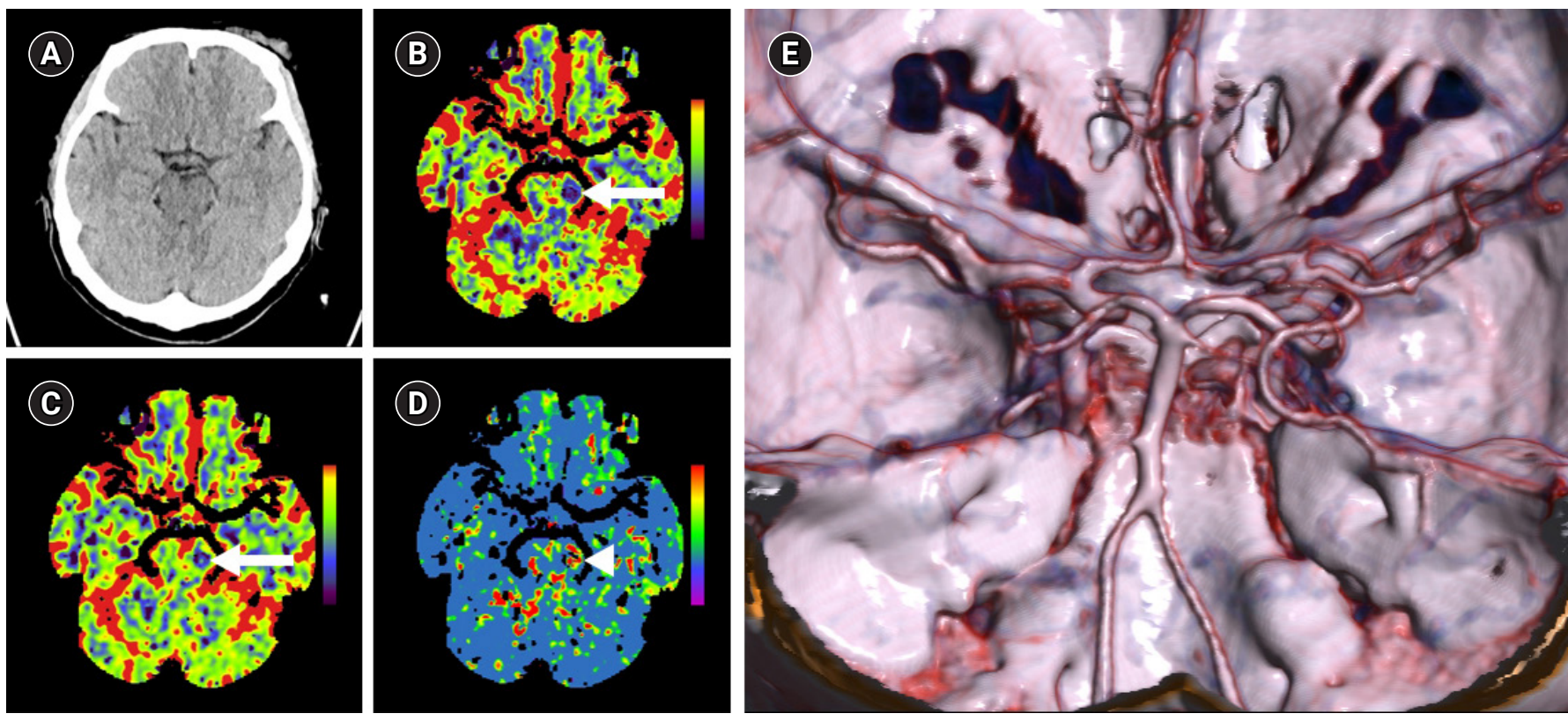

Fig. 1. Brain computed tomography (CT) and CT angiography were performed immediately after arrival at the emergency room. (A) There was no remarkable finding on brain CT. (B) Cerebral blood flow and (C) cerebral blood volume map shows decreased perfusion of the left midbrain (arrows). (D) Mean transit time map shows a prolongation within the same region (arrowhead), indicative of core infarct in the left midbrain. (E) There were no stenosis or occlusion on basilar artery and the other vessels. 
thrombosis, the antiplatelet agent was changed to an anticoagulant agent (dabigatran $300 \mathrm{mg}$ /day). Transcranial Doppler was performed but showed normal results. Neither the bubble test nor the transesophageal echocardiography (TEE) was could not be performed because valsalva maneuver could not be performed due to stuporous mentality. Protein $\mathrm{C}$ activity was rechecked 3 months later and the result was restored to normal as $64 \%$.

\section{DISCUSSION}

As improving understand for stroke pathophysiology and achieving advances in imaging, vague defined entity of cryptogenic stroke was reassessed as ESUS to improve the efficacy of secondary stroke prevention by International Working Group of Neurologists in 2014 [1]. ESUS is known to account for an average of
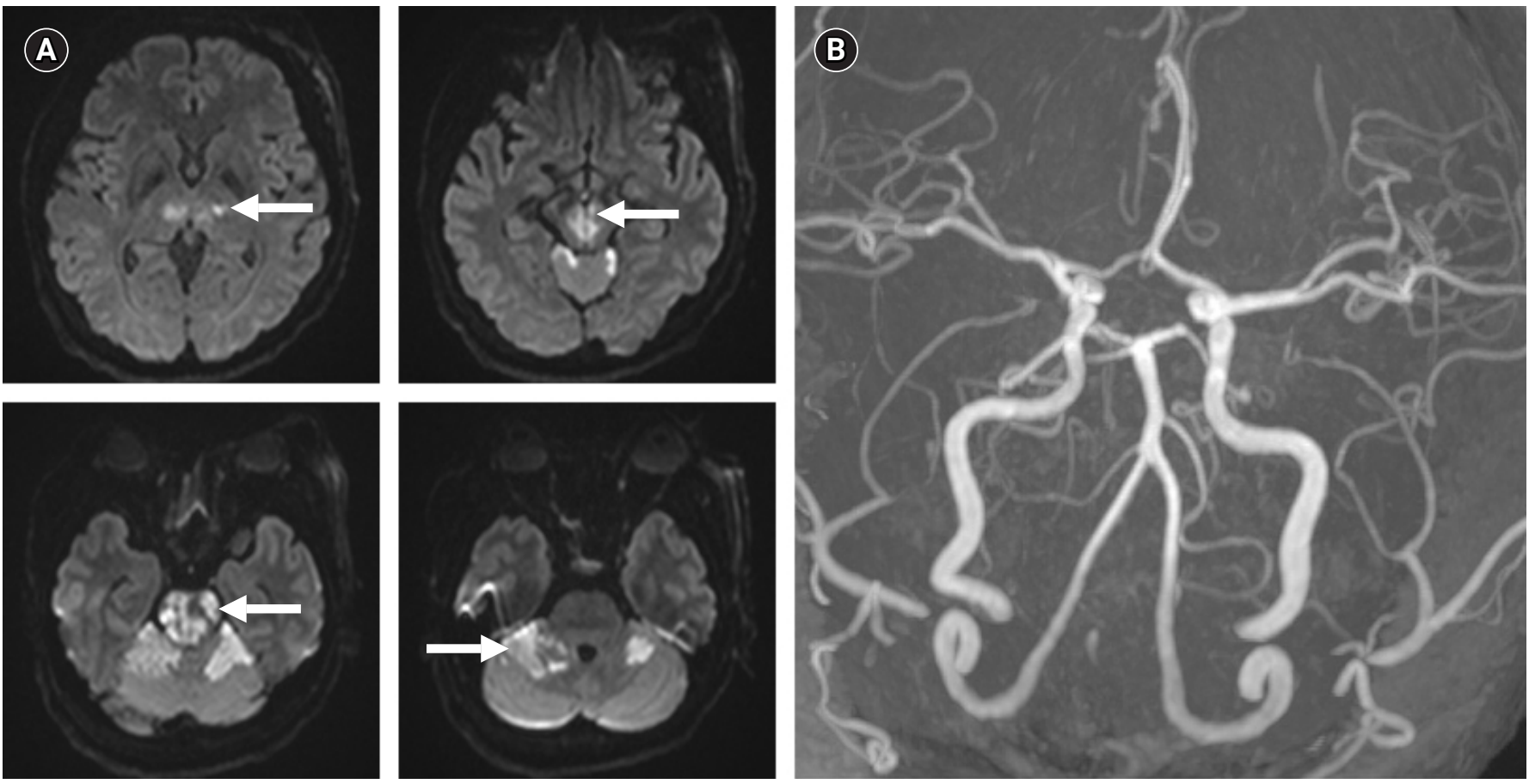

Fig. 2. (A) Diffusion-weighted imaging, performed after 1 day from symptom onset, showed acute ischemic lesion in the bilateral thalamus, midbrain, pons and right cerebellum (arrows). (B) Magnetic resonance angiography which was performed after 1 day from symptom onset, showed no stenosis or occlusion.
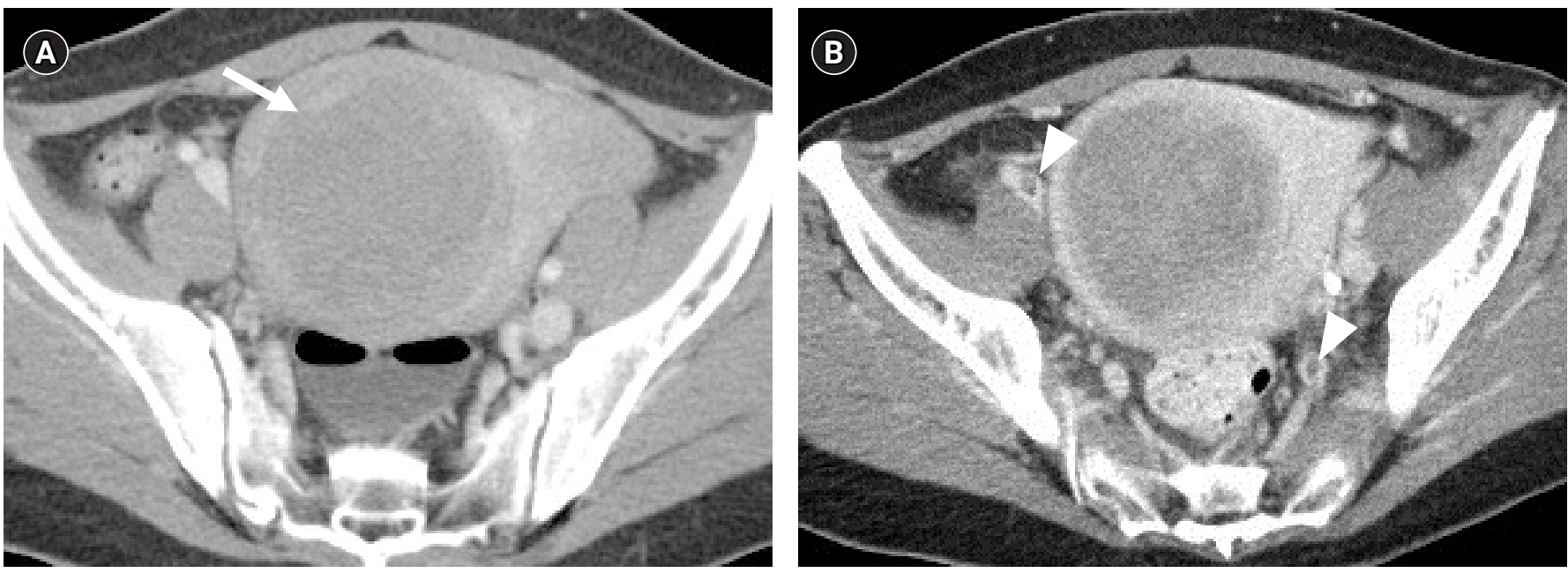

Fig. 3. (A) There was a $7.5 \mathrm{~cm}$ uterine myoma with several small myomas in pelvic cavity (arrow). (B) Femoral computed tomographic angiography reveals contrast filling defects in the right external iliac vein, left internal iliac vein (arrowheads). 
$17 \%$ according to a study of ischemic stroke patients [1]. The most common cause of ESUS patients is paroxysmal atrial fibrillation. Recently, various studies have revealed a strong relationship with PFO and ESUS [2]. However, there are various causes such as vascular atherosclerotic ulcer, $\mathrm{PFO}$, and embolism associated with cancer [3].

Searching the cause of embolus in ESUS is very important for establishing secondary prevention plan. Although systemic thromboembolism to lung, kidneys, spleen, and brain is a very rare complication of uterine myoma, there are several reports that uterine myoma can cause systemic embolism. One suggested mechanism is that uterine myoma may cause severe anemia and reactive thrombocytosis, leading to arterial thrombosis and thus recurrent cerebral infarct in patient without no $\mathrm{PFO}$ and no thrombus in pelvic organ $[4,5]$. Other suggested mechanism is that deep venous thrombosis due to compression of the lower extremity vein caused by a huge uterine myoma may enter systemic circulation through PFO and cause relapsing paradoxical cerebral embolism [6-8].

Because there was the presence of multiple thrombosis in both lower extremity veins without anemia or coagulopathy in this case, we thought the paradoxical embolism of deep vein thrombosis by large uterine myoma through PFO is the etiology of this case rather than anemia and coagulopathy although she could not confirm the PFO by performing TEE or bubble test due to stuporous mentality. Failure to identify the right-to-left shunt is a limitation of this case. In here, we report this case because uterine myoma in a middle-aged woman can be thought to be the etiological cause that can contributor to deep vein thrombosis, and it is necessary to pay attention as the etiology of ESUS.

\section{ARTICLE INFORMATION}

\section{Conflict of interest}

No potential conflict of interest relevant to this article.

\section{ORCID}

Hyun Joon Lee, https://orcid.org/0000-0001-6295-182X

Dong Hoon Shin, https://orcid.org/0000-0002-1103-1983

\section{Author contributions}

Conceptualization: LHJ and SDH. Data curation \& Formal analysis: LHJ and SDH. Investigation: LHJ and SDH. Methodology: LHJ and SDH. Project administration: LHJ and SDH. Resources: LHJ and SDH. Supervision: LHJ and SDH. Validation: LHJ and SDH. Visualization \& Writing-original draft: LHJ and SDH. Writing-review editing: LHJ and SDH.

\section{REFERENCES}

1. Hart RG, Catanese L, Perera KS, Ntaios G, Connolly SJ. Embolic stroke of undetermined source: a systematic review and clinical update. Stroke 2017;48:867-72.

2. Wu LA, Malouf JF, Dearani JA, Hagler DJ, Reeder GS, Petty GW, et al. Patent foramen ovale in cryptogenic stroke: current understanding and management options. Arch Intern Med 2004;164:950-6.

3. Gladstone DJ, Spring M, Dorian P, Panzov V, Thorpe KE, Hall J, et al. Atrial fibrillation in patients with cryptogenic stroke. N Engl J Med 2014;370:2467-77.

4. Higuchi E, Toi S, Shirai Y, Mizuno S, Onizuka H, Nagashima Y, et al. Recurrent cerebral infarction due to benign uterine myoma.J Stroke Cerebrovasc Dis 2019;28:e1-2.

5. Akarsu S, Tekin L, Çarlı AB, Güzelküçük Ü, Yılmaz A. Stroke due to anemia after severe menstrual bleeding caused by a uterine myoma: the title is self-explanatory. Acta Neurol Belg 2013; 113:357-8.

6. Toru S, Murata T, Ohara M, Ishiguro T, Kobayashi T. Paradoxical cerebral embolism with patent foramen ovale and deep venous thrombosis caused by a massive myoma uteri. Clin Neurol Neurosurg 2013;115:760-1.

7. Nakamura S, Tokunaga T, Yamaguchi A, Kono T, Kasano K, Yoshiwara $\mathrm{H}$, et al. Paradoxical embolism caused by ovarian vein thrombosis extending to inferior vena cava in a female with uterine myoma. J Cardiol Cases 2018;18:207-9.

8. Srettabunjong S. Systemic thromboembolism after deep vein thrombosis caused by uterine myomas. Am J Forensic Med Pathol 2013;34:207-9. 